\title{
Simple and Efficient Numerical Evaluation of Near-Hypersingular Integrals
}

\author{
Patrick W. Fink, Member, IEEE, Donald R. Wilton, Fellow, IEEE, Michael A. Khayat, Member, IEEE
}

\begin{abstract}
Simple and efficient numerical procedures for evaluating the gradient of Newton-type potentials are presented. Convergences of both normal and tangential components of the gradient are examined. The convergence of the vector potential is also examined, and it is shown that the scheme for handling near-hypersingular integrals also is effective for the nearly singular potential terms.
\end{abstract}

Index Terms - Numerical integration, Integral equations, Computation theory .

\section{INTRODUCTION}

$\mathrm{R}$ ecently, significant progress has been made in the handling of singular and nearly-singular potential integrals that commonly arise in the Boundary Element Method (BEM) [1-3]. To facilitate object-oriented programming and handling of higher order basis functions, cancellation techniques are favored over techniques involving singularity subtraction. However, gradients of the Newtontype potentials, which produce hypersingular kernels, are also frequently required in BEM formulations. As is the case with the potentials, treatment of the near-hypersingular integrals has proven more challenging than treatment of the limiting case in which the observation point approaches the surface. Historically, numerical evaluation of these nearhypersingularities has often involved a two-step procedure: a singularity subtraction to reduce the order of the singularity, followed by a boundary contour integral. Departure from object-oriented philosophy and heightened complexity in the handling of higher order basis functions are even more problematic in this context. Thus, there is ample need for cancellation-type techniques for efficient numerical evaluation of the gradient of the potential.

Progress in the development of efficient cancellation-type

P. W. Fink is with NASA Johnson Space Center, Houston, TX, 77058, USA (email: patrick.w.fink@nasa.gov)

D. R. Wilton is with the Deptartment of Electrical and Computer Engineering, University of Houston, Houston, TX, 77204-4793, USA. (email: wilton@uh.edu)

M. A. Khayat is with NASA Johnson Space Center, Houston, TX, 77058, USA (email: michael.a.khayat@nasa.gov) procedures for the gradient potentials was recently presented $[2,4]$. The approach in deriving these procedures closely parallels that which led to the so-called "Arcsinh" transformation [1]. That is, to the extent possible, a change of variables is chosen such that the Jacobian of the transformation cancels the singularity. However, since the gradient kernel involves singularities of different orders, we extend the approach to require that the transformation reduces the order of the singularity and leaves remaining terms that are analytic. The terms "normal" and "tangential" are used herein with reference to the source element.

For the singular potential terms, the integral is defined and well-behaved when the observation point is contained within the source element. However, for the gradient potential terms we must be content with numerical evaluation in which the observation point remains some finite, but arbitrarily small, distance, $d$, above the source element. Procedures for extracting the normal component of the gradient in the limit as the observation point approaches the source element are well known and are not discussed herein.

The work in [2] presents the desired singularity order reduction for the gradient terms and enables extremely efficient computation of the normal gradient component as the distance $z$ tends toward zero. However, convergence as presented in [2] is strongly dependent upon $z$. Also, the tangential gradient components are not addressed in [2], primarily because the techniques therein do not permit noticeably efficient evaluation. Also, since computational formulations often involve the numerical evaluation of the potentials in addition to their gradients, it is highly desirable that a single integration procedure efficiently handle both. Techniques to extend and improve the methods in [2] were recently presented [4] and are the primary subject of this paper.

\section{INTEGRALS WITH NEAR-HYPERSINGULARITIES}

\section{A. Theory}

In this section, the transformations previously presented [2, 4] are reviewed. Further modifications to improve convergence, of both the normal and tangential gradient components, are established. Cartesian and spherical coordinates are used herein to simplify notation and to readily 
convey mathematical behavior. In practice, use of simplex coordinates defined on the parent element is often preferred, and conversion of quadrature points on sub-elements follows simple procedures previously outlined [1].

In order to simplify the presentation and evaluation of the proposed methods, we consider the following integral representative of the actual integrals with respect to the convergence of numerical integration:

$$
I=\int_{D} b\left(r^{\prime}\right) \nabla\left(\frac{e^{-j k R}}{4 \pi R}\right) d \mathcal{D},
$$

where $R=\left|\mathbf{r}-\mathbf{r}^{\prime}\right|$ is the distance between an observation point at $\mathbf{r}$ and source points on triangular domains $\mathcal{D}$. Here, the function $b$ is intended to capture the behavior of the basis function. Following previous approaches [1-5], a nearby observation point is projected onto the surface containing the source parent triangular element. The parent triangle is then split into three subtriangles about the projected observation point. The geometry of a typical subtriangle and its local rectangular coordinate system with origin at the projected observation point is shown in Fig. 1.

The general form of the transformed integral over the subtriangle is given by

$$
\begin{aligned}
& \int_{0}^{h} \int_{y \cot \phi_{L}}^{y \cot \phi_{U}} g(x, y) d x d y \\
& \quad=\int_{v_{L}}^{v_{U}} \int_{u_{L}}^{u_{U}} g[x(u, v), y(u, v)] \mathcal{J}(u, v) d u d v,
\end{aligned}
$$

where $g(x, y)$ is a product of the basis and the Green's function. Ideally, the transform's Jacobian $\mathcal{J}$ would exactly cancel the singular (static) part of the kernel for constant source densities, and the subtriangle would map into a rectangular domain such that repeated Gauss-Legendre integration of low order may be used. A summary of two transformations previously investigated $[2,4]$ is given in Table 1 , followed by a synopsis of their properties.

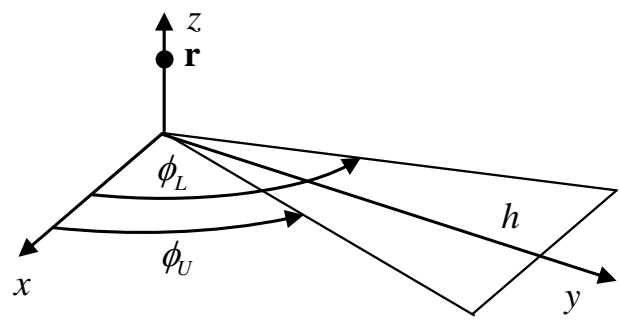

Fig. 1. Subtriangle geometry

Note that both transformations result in terms, $\rho$, which arise in the linear basis function components and in the $x$ - and $y$ components of the vector, $\mathbf{R}$. These terms, which are nonanalytic in $v$, must be handled carefully, and there are other detractions associated with the $\mathrm{R}^{3}$ transformation to be noted. It is extremely efficient for evaluating the static normal component of the gradient $(k=0)$. However, it is very poor in capturing any of the other terms [2]; that is, $\rho$ and higher powers of $R$, due to the near-singularities introduced in these terms by the transformation. Moreover, we present here a means, using the $\mathrm{R}^{2}$ transformation, to handle the normal component of the gradient with the same high efficiency, but none of the detractions, characterizing the $\mathrm{R}^{3}$ method. For this reason, the $R^{3}$ is not considered further in this paper. The $R^{2}$ transformation results in smooth functions, but can result in slow convergence when the ratio of the end boundary value of $v$ to the initial boundary value of $v$ is large.

TABLE 1: SUMMARY OF TRANSFORMATIONS

\begin{tabular}{|l|l|l|l|}
\hline & TRANSFORMATION & $\mathcal{J}(u, v)$ & INTEGRATION LIMITS \\
\hline Radial-Angular & $u=\phi$ & $R^{2}$ & $u_{\mathrm{L}, U}=\ln \tan \frac{\phi_{\mathrm{L}, U}}{2}$ \\
$\mathrm{R}^{2}$ & $v=\ln R$ & $v_{L, U}=\ln |z|, \frac{1}{2} \ln \left(z^{2}+(h \cosh u)^{2}\right)$ \\
\hline $\begin{array}{l}\text { Radial-Angular } \\
\mathrm{R}^{3}\end{array}$ & $u=\phi$ & $\frac{-R^{3}}{|z|}$ & $\begin{array}{l}u_{L, U}=\phi_{L, U} \\
v_{L, U}=1, \frac{|z|}{\sqrt{z^{2}+(h / \sin u)^{2}}}\end{array}$ \\
\hline
\end{tabular}

Radial-Angular $\mathrm{R}^{2}$ :

- Introduces $\mathrm{R}^{2}$ in the Jacobian to reduce singularities in $R$

- $R$ becomes a smooth function of the integration variable, v

- Introduces terms, $\rho$, that are non-analytic at $R=z$

Radial-Angular $\mathrm{R}^{3}$ :

- Introduces $\mathrm{R}^{3}$ in the Jacobian to reduce singularities in $\mathrm{R}$

- Exactly integrates the normal gradient component of the static potential with one sample point per subtriangle

- Introduces nearly-singular components, $(|z| / v)^{m}$.

- Introduces terms, $\rho$, that are non-analytic at $R=z$

To improve the convergence using the $\mathrm{R}^{2}$ transformation, we consider the introduction of a disk of radius $\rho_{0}$ centered at the projection point as shown in Figure 2, along with the three subtriangles. Since the transformations listed in Table 1 already employ radial and angular boundaries, inclusion of the disk element is seamless. The introduction of this fourth subelement provides three important advantages. First, the azimuthal variation of the gradient integrand can be integrated exactly with only three sample points, assuming linear basis functions (completeness order $p=0$ ), or with two sample points for constant basis functions. The 3-point azimuthal rule comprises three equally-spaced angles and equal weights - a Riemann sum; e.g., $\left\{\phi_{i}\right\}=2 \pi\{1 / 6,1 / 2,5 / 6\} \quad$ and $\left\{w_{i}\right\}=\{1 / 3\}$. It exactly integrates the set of functions $\left\{f_{i}\right\}=\{1, \sin \phi, \cos \phi, \sin 2 \phi, \cos 2 \phi\} \quad$ over the circle. For potentials, only two sample points are required for exact integration of the azimuthal component of the integrand with linear bases, or one sample point for constant bases. The second key advantage pertains to the non-analyticity of $\rho$. Since the azimuthal components are integrated exactly, all odd powers of $\rho$, which are non-analytic and are multiplied by odd powers of the sine or cosine functions, are entirely eliminated over the disk. 


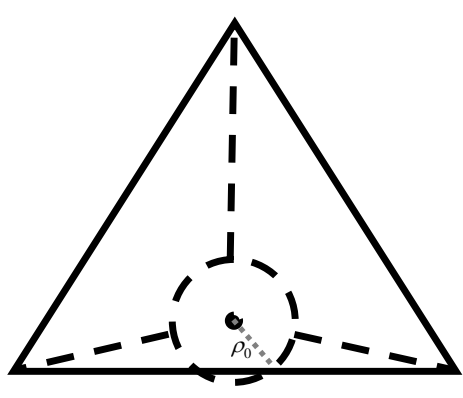

Figure 2. Disk sub-element centered at projection point.

The third key advantage pertains to the radial variation of the integrand, and we now show that accurate integration of the radial terms over the disk can also be achieved with great efficiency. Upon application of the Radial-Angular $\mathrm{R}^{2}$ transformation, the integrand of (1) can be written as

$$
i_{G}=b\left(\mathbf{r}^{\prime}\right)(1+j k R) e^{-j k R} \frac{\mathbf{R}}{R},
$$

in which dependencies upon the variables of integration have been suppressed. Since all terms with odd powers of the cylindrical radius, $\rho$, are eliminated upon integration over the disk, the integrand is reduced to factors with the following powers of R:

$$
\mathscr{F}=\left\{R^{-1}, 1, R,\right\}\left\{1-j k R-k^{2} R^{2}-\ldots\right\},
$$

where the complex exponential has been expanded. We now consider integration of a single term of power $m$ and make use of a normalized variable of integration, $\lambda$,

$$
I_{m}=\int_{v_{l}}^{v_{u}} e^{m v} d v=\Delta \int_{0}^{1} e^{m\left(\Delta \lambda+v_{l}\right)} d \lambda,
$$

where

$$
\begin{gathered}
m=-1,0, . . ; v_{l, u}=\ln \left(z, R_{0}\right), R_{0}=\sqrt{\rho_{0}^{2}+z^{2}}, \\
\text { and } \Delta \equiv v_{u}-v_{l} .
\end{gathered}
$$

Equation (5) can then be rewritten

$$
I_{m}=\Delta \int_{0}^{1} z^{m} e^{m \lambda \ln \alpha} d \lambda
$$

where

$$
\alpha \equiv R_{0} / z
$$

We now consider means to numerically integrate the dominant terms in (4) efficiently. We assume that the disk radius is small relative to a wavelength, and thus address only the constant and linear components in the expansion of the phase term. The set of $R$-dependent terms is then reduced to

$$
\mathscr{F}=\left\{e^{-\ln (\alpha) \lambda}, 1, e^{\ln (\alpha) \lambda}, e^{2 \ln (\alpha) \lambda}\right\} .
$$

For efficient integration of these terms over the disk, we employ a 2-point quadrature rule, which integrates the terms in (8) exactly, providing that (7) is satisfied. Since (7) pertains to a specific ratio of radius to $z$, we create a table of 2-point quadrature rules to cover the desired range of $\alpha \mathrm{s}$. Each table entry corresponds to a specific value of $\alpha$, and we choose the entry, $\tilde{\alpha}$, nearest to the desired value given by (7). We then modify the disk radius to match the selected table entry,

$$
\tilde{R}_{0}=\mathrm{z} \tilde{\alpha},
$$

so that all terms represented in (8) are integrated exactly over the disk.

If the table has a sufficient number of entries, the modified disk radius is sufficiently close to the desired radius that there are no adverse consequences, as is demonstrated in the next section. For a table accommodating values $1<\alpha<10^{5}$, a maximum ratio $\left(z / \rho_{0}\right)_{\max }=2$, and a deviation from the desired disk radius below ten percent, the total table size is 103 quadrature rules.

It remains to determine the desired value of disk radius. Since integration over the disk is particularly efficient, and integration external to the disk is prone to effects of the nonanalyticity of $\rho$ at $R=z$, larger values of disk radius appear advantageous. Extension of the disk region beyond the subtriangle boundaries (as shown in Fig. 2) is readily accommodated and requires no additional considerations. In this case, outlying regions of the disk acquire a negative weight due to the reversal of integration between the circular boundary and the outer subtriangle edge. Initial trials, however, reveal no significant advantages to extending the radius beyond the triangle boundaries, and it is convenient to set the disk radius

$$
\rho_{0}=h_{\min },
$$

the minimum subtriangle height (Figure 1).

\section{B. Results}

The triangle shown in Figure 3 is used as a test case for three different heights, $z$, of the observation point: $10^{-6} \mathrm{~m}, 0.01$ $\mathrm{m}$, and $0.2 \mathrm{~m}$. For all but one case, as noted below, the disk radius equals $0.1 \mathrm{~m}$. The wavelength is $10.0 \mathrm{~m}$ so that the longest edge is 0.1 wavelengths. The basis function variation is chosen as $b=x+y$ (Figure 3 ), and the $\mathrm{R}^{2}$ transformation is used for all trials.

Except as noted below, the specialized quadrature schemes presented above for the $u$ - and $v$-directions are applied for integration over the disk region. The disk radius is set to 0.1 $\mathrm{m}$, except for one case to demonstrate insensitivity to this parameter. Over the truncated triangular regions, GaussLegendre is used to integrate the transformed integrals along both radial and angular directions. Each truncated subtriangle was analyzed individually to determine near-optimal sampling ratios along the two directions $(u, v)$ in order to achieve a near monotonic convergence for both the normal and tangential gradient components. Convergence trends of the normal and tangential components are shown in Figures 4 and 5, respectively, with number of sample points indicating the combined total for disk and all three subtriangle regions.

As seen in Figure 4, accurate integration of the normal gradient component is extremely efficient as $z$ tends to zero. 
The crossed square symbols in this figure denote disk quadrature schemes with fewer than six sample points. In the extreme case, just 1 sample point (in the disk) is used for the whole source triangle. In contrast to previous work demonstrating great accuracy with 1-2 points [2], the introduction of the disk permits continued convergence of the normal component with more sample points, as well as convergence of the tangential component. The cross symbols in Figure 4 represent use of a disk radius $\rho_{0}=0.09$, and it can be seen that the effects are minimal. As $z$ becomes sufficiently large, the $v$-components can be integrated efficiently over the disk with a two-point Gauss-Legendre scheme. For example, in Figures 4 and 5, for the case $z=0.2$ and the range of sample points shown, very little difference was observed when the $v$-components were integrated with a two-point Gauss-Legendre scheme compared to the two-point specialized quadrature described in the preceding section.

$(-0.5,-0.5)$

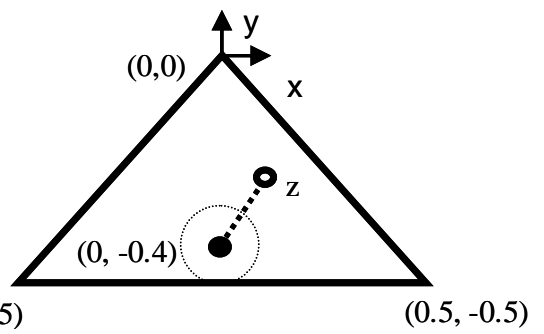

Figure 3. Triangle geometry for trials.

Figure 6 shows the convergence results for the potential using the same quadrature rules applied to the gradient. The crossed square symbols indicate trials in which the $3 \times 2$ quadrature rule over the disk is replaced by a $3 \times 3$ rule in order to assess eventual limitations due to the truncation of the phase term implicit in (8). It should be noted that one could improve the convergence for the potential by employing the same concepts (i.e., specialized quadratures over the disk) and optimizing specifically for the potential. For example, only two points are required for the $u$-integration over the disk, and the term with the negative exponent in (8) would be replaced by the next positive integer exponent. Further work is required to compare this approach with another recent advance for integration of 1/R-type singularities [3].

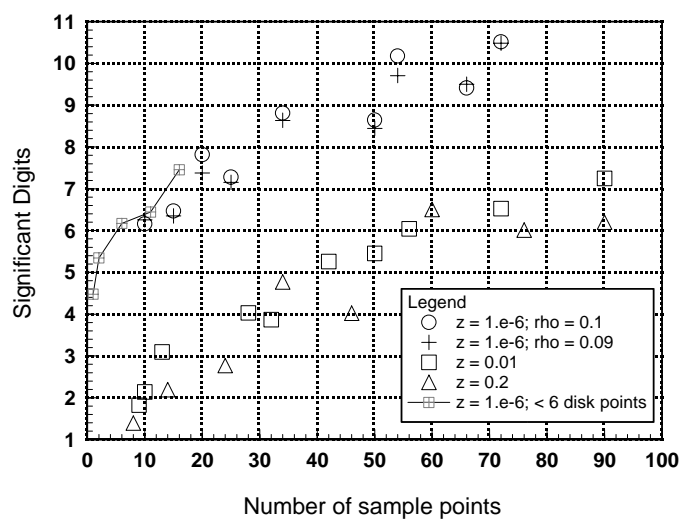

Figure 4. Convergence of normal gradient component.

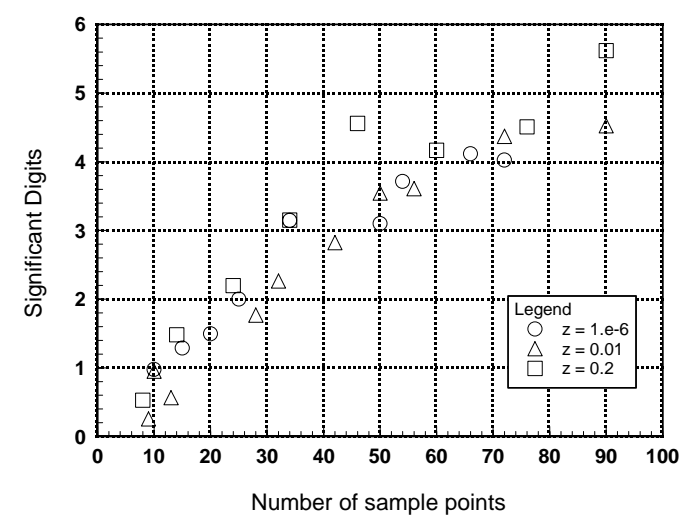

Figure 5. Convergence of tangential gradient component.

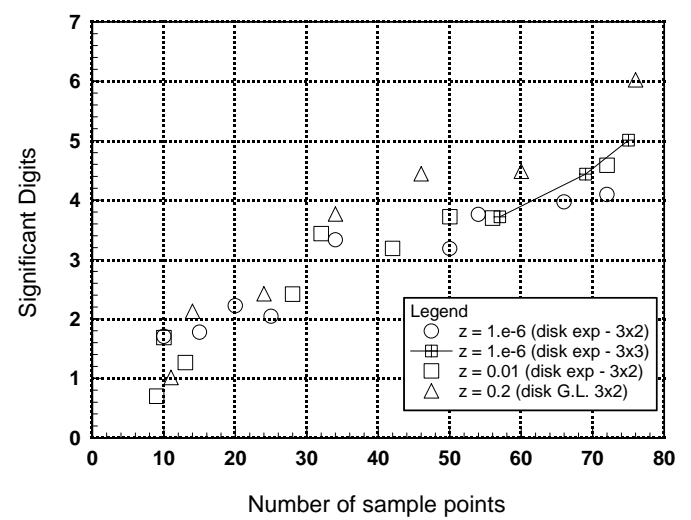

Figure 6. Convergence of the potential using the gradientbased quadrature rules.

\section{REFERENCES}

[1] M. A. Khayat and D. R. Wilton, "Numerical Evaluation of Singular and Near-Singular Potential Integrals," IEEE Trans. Antennas Propagat.., vol. 53, no. 10, pp. 31803190, Oct. 2005.

[2] P. W. Fink, D. R. Wilton, and M. A. Khayat, "Issues and Methods Concerning the Evaluation of Hypersingular and Near-Hypersingular Integrals in BEM Formulations", Proc. ICEAA, pp. 861-864, Torino, Italy, 2005.

[3] M. A. Khayat, D. R. Wilton, and P. W. Fink, “A Comparison of Transformations for the Evaluation of Singular and Near-Singular Potential Integrals", IEEE Antennas Wireless Propag. Lett., submitted".

[4] P.W. Fink, D. R. Wilton, and M. Khayat, "Refinement of Methods for Evaluation of Near-Hypersingular Integrals in BEM Formulations", URSI National Radio Science Meeting, Albuquerque, N.M., 2006.

[5] M. G. Duffy, "Quadrature over a pyramid or cube of integrands with a singularity at a vertex," SIAM J. Num. Anal., vol. 19, issue 6, pp. 1260-1262, 1982. 
